\title{
Biopolymer/Gold Nanoparticles Composite Plasmonic Thermal History Indicator to Monitor Quality and Safety of Perishable Bioproducts
}

\author{
Yi-Cheng Wang ${ }^{\ddagger}$ Lin $\mathrm{Lu}^{\ddagger}$, and Sundaram Gunasekaran* \\ Department of Biological Systems Engineering \\ University of Wisconsin-Madison \\ Madison, WI 53706, USA
}

\$ Authors contributed equally

* Corresponding author e-mail: guna@wisc.edu 


\begin{abstract}
Quality and safety of perishable products such as foods, pharmaceutics, and biologicals is a constant concern. We have developed a plasmonic thermal history indicator (THI) taking advantage of the localized surface plasmon resonance of gold nanoparticles (AuNPs) synthesized in situ in alginate, a natural polysaccharide. The color of the THIs becomes more intense with increased storage temperature and/or duration, with the color changing from grey to red with time of exposure at high temperature $\left(40^{\circ} \mathrm{C}\right)$. The results suggest that decreasing viscosity with increasing number of AuNPs being synthesized in the system, along with aggregation of newly synthesized AuNPs onto larger ones and their settling are potentially responsible for the distinct color change observed. The use of alginate in the THIs also facilitates fabricating them as solid hydrogel matrices by adding divalent calcium ions. This alginate-AuNPs THI system is tunable by altering its composition to suit different time-temperature monitoring scenarios and the color-change reaction is irreversible. The THI provides a convenient, reliable, safe, and inexpensive means for tracking the thermal history of perishable products without the need for a read-out device.
\end{abstract}

Keywords: Localized surface plasmon resonance (LSPR), food safety, alginate, colorimetric sensors, time temperature indicator, hydrogel 


\section{Introduction}

Many food products, pharmaceutics, and biologicals such as cord blood, stem cells, enzymes, antibodies are sensitive to temperature and easily deteriorate over time (Derwood 2007; Louis et al. 2012; Zheng and Janis 2006). The Food and Drug Administration (FDA) has defined a class of foods that requires time and temperature control for their safety as TCS (time and temperature control for safety) foods, which include a wide range of natural, processed, raw, and cooked foods of both animal and plant origin that are prevalent in our daily diet (Food Code 2013, FDA). Therefore, the shelf life of these perishable goods is very critical for the consumers.

Generally, most perishable products are marketed with a sell-by or use-by date printed on the product package. However, these expiration dates are typically estimated based on several simplified assumptions, including that the product is always held at safe temperature, which is hardly the case. In addition, uncontrolled temperature fluctuations during handling, distribution, and storage are inevitable (Taoukis and Labuza 1989; Wang et al. 2015a). Since the thermal history of the products is a major factor that affects the product quality and safety, the credibility of existing expiration date labeling to assure product safety is questionable.

To address this issue, time-temperature indicators (TTIs), which can help monitor time- and temperature-dependent changes in product quality and/or safety have been developed. One major type of TTI is electronics-based, including thermal sensor, temperature logger and/or microprocessor (Wang et al. 2015a). However, these digital devices are generally expensive and complicated therefore not applicable to individual packages or products. Colorimetric/optical TTIs, on the other hand allow prompt monitoring without the need for read-out devices or trained personnel to conduct the test, serve as cost-effective and user-friendly alternatives. Accordingly, dye- and enzyme-based TTIs have been developed (Galliani et al. 2015; Kim et al. 2012; Pereira Jr et al. 2015; Wanihsuksombat et al. 2010). However, TTIs that utilize the distinct and advantageous properties of nanomaterials are still under active development (Wang et al. 2015c; Zhang et al. 2013). An improvement over the traditional TTI is the thermal history indicator (THI) we have designed, where the THI can closely track the path of temperature variation during storage and handling, not just the cumulative effect of the time-temperature change as TTIs do. We have shown that the size and shape of gold nanoparticles (AuNPs) that evolve in gelatin under different time and temperature scenarios exhibit different colors, which could be used as THI (Lim et al. 2012; Wang et al. 2015c). The alginate-based THIs reported herein has several advantages over the gelatin-based THIs such as higher color-change sensitivity, wider 
range of temperature monitoring, and the ability to prepare solid-like THI matrices. Also, the gelatin/AuNPs were designed specifically for low-temperature storage. Furthermore, to the best of our knowledge, little published information is available on the interaction between biopolymer and in situ synthesized nanoparticles, especially with respect to change in solution viscosity and its effect on time- and temperature-dependent change in localized surface plasmon resonance (LSPR) of AuNPs. Heli et.al (Heli et al. 2016) developed a plasmonic nanopaper sensor which undergoes color change of silver nanoparticles embedded in bacterial cellulose in response to the presence of some volatile compounds released by foods upon spoilage. THIs we describe herein can continuously track the thermal history of the product, which is closely related to product quality and/or safety given the well-known temperature-dependent microbial and biochemical kinetics throughout the product life, rather than simply detect the end-of-life spoiled state of the product only when it is reached. It is well known that inappropriate storage history confers adverse effects on quality of perishable products, even if no detectable sign of spoilage is present. For instance, even brief and unintended exposures to high temperatures can promote deteriorative processes. Furthermore, foods that have undergone deteriorative conditions but do not yet exhibit detectable signs of spoilage, may harm immunosuppressed populations, which include children and elderly.

Herein we report alginate/AuNPs-based THIs. Alginate is a non-toxic, biocompatible, and inexpensive polysaccharide; it is widely used in pharmaceutical and biomedical applications (Boland et al. 2007; Xu et al. 2010). The AuNPs are highly biocompatible and possess distinct surface plasmon properties (Bagci et al. 2015; Ghosh et al. 2008; Guan et al. 2015; Lu et al. 2016; Wang et al. 2015b; Wei et al. 2015; Zhang et al. 2015). Furthermore, our results indicate, contrary to Einstein-Batchelor viscosity law, that in situ synthesis of AuNPs in alginate solution lowers its viscosity, as had been reported for some other polymeric solutions with nanoparticles addition (Jain et al. 2008; Mangal et al. 2015). The change in viscosity of a biopolymer solution could potentially affect the size and/or shape of AuNPs being synthesized in situ (Wang et al. 2015c), hence change their LSPR and the corresponding color. Experiments show that our alginate/AuNPs THIs, owing to the LSPR of the evolving AuNPs, exhibit visibly distinguishable color change, which is indicative of the thermal history of the products being tracked. Further, the propensity for alginate to gel with the addition of calcium ions allows fabrication of alginate/AuNPs THIs into solid hydrogel matrices. Hence, the alginate/AuNPs system can serve 
as convenient, reliable, safe, and inexpensive THIs for monitoring quality and/or safety of perishable bioproducts.

\section{Experimental}

\subsection{Materials}

Hydrogen tetrachloroaurate (III) trihydrate $\left(\mathrm{HAuCl}_{4} \cdot 3 \mathrm{H}_{2} \mathrm{O}\right)$ and alginic acid sodium salt were purchased from Acros Organics (Morris Plains, NJ, USA). Calcium chloride dehydrate $\left(\mathrm{CaCl}_{2} \cdot 2 \mathrm{H}_{2} \mathrm{O}, 99 \%\right)$ was obtained from Fisher Scientific (Rockford, IL, USA). All chemicals were used as received and all aqueous solutions were prepared in Millipore water (resistivity >18 $\mathrm{M} \Omega \cdot \mathrm{cm})$ unless mentioned elsewhere.

\subsection{THI Preparation}

For fabrication of typical alginate/AuNPs THIs, sodium alginate solution $\left(0.01 \mathrm{~g} \cdot \mathrm{mL}^{-1}\right)$ is first prepared by stirring its powder in water at $90{ }^{\circ} \mathrm{C}$ for $30 \mathrm{~min}$. Gold precursor solution $(0.1 \mathrm{v} / \mathrm{v}$, $10 \mathrm{mM}$ ), prepared by dissolving $\mathrm{HAuCl}_{4}$ in water, is mixed with the sodium alginate solution and heated at $90^{\circ} \mathrm{C}$ for 2.5 min under constant stirring. One milliliter of the mixture is then placed in $1.5 \mathrm{~mL}$ cuvettes, which serve as the THIs. THI hydrogel matrices were prepared by adding $100 \mu \mathrm{L}$ of $1 \%$ calcium chloride solution into the alginate- $\mathrm{HAuCl}_{4}$ solution mixture.

\subsection{Characterization}

The absorption spectra of the THI solution samples were measured with Lambda 25 ultraviolet-visible (UV-Vis) spectrophotometer (PerkinElmer). Attenuated total reflectance-Fourier transform infrared (ATR-FTIR) spectra were performed with Spectrum 100 FT-IR spectroscopy (PerkinElmer). The morphology of the nanoparticles was imaged using FEI Tecnai T12 transmission electron microscope (TEM) operating at $120 \mathrm{kV}$. For the TEM sample preparation, typically, one drop of sample was placed on a carbon-coated copper grid and excess solution was removed by wicking with filter paper. The grid was then allowed to dry at room temperature (RT).

ImageJ (Wayne Rasband, National Institutes of Health, USA) was used to determine the color change in the THIs exposed to different temperatures. All photographs of THI samples were taken in the same conditions (same camera settings, time, location and lighting, etc.), and were first 
calibrated using NIP2, the graphical user interface of VIPS open source image processing system, before being imported to ImageJ for color difference measurement.

The X-ray diffraction (XRD) pattern of AuNPs was obtained on an X-ray diffractometer (Bruker D8 Discover, Karlsruhe, Germany) with $\mathrm{Cu} \mathrm{K \alpha}$ radiation at $50 \mathrm{kV}$ tube voltage and 1.0 mA tube current. XPS analysis was performed by Thermo K-alpha XPS. For XRD and X-ray photoelectron spectroscopy (XPS), $0.5 \mathrm{~mL}$ of the sample solution was placed on a glass slide and allowed to dry at RT before measurement. The viscosity was investigated using Kinexus ultra+ rheometer (Malvern Instruments, UK) equipped with C25 bob-and-cup geometry, with a gap size of $1 \mathrm{~mm} .17 .25 \mathrm{~mL}$ of sample (at $25^{\circ} \mathrm{C}$ ) was used for viscosity measurement over a shear rate range of 0.1 to $1000 \mathrm{~s}^{-1}$.

\section{Results and Discussion}

\subsection{Time- and Temperature-dependent color change}

Like most chemical reactions, the in situ synthesis of AuNPs is dependent on temperature and time. Thus, the temperature history of the sample affects the size and shape evolution of AuNPs, which gives rise to visible color change signal. Therefore, the observed color of the THIs can be correlated with their thermal history. Since the quality and safety of perishable bioproducts are dependent on their thermal history, color of the THI can be used to indicate the quality and safety of the products being monitored.

During the synthesis of THIs, alginate serves as a mild reducing reagent and reduces the gold ions to gold atoms, which then aggregate to form AuNPs. The color of the as-prepared THIs is translucent light grey (Fig. 1a), which suggests the presence of AuNPs and is confirmed by TEM (Fig. S1a). The prolonged heating leads to deeper grey color (Fig. S1b), indicating additional synthesis of AuNPs. These results also imply that AuNPs synthesis reaction is strongly thermal history dependent.

We subjected THIs to four storage temperatures $\left(-20^{\circ} \mathrm{C}, 0^{\circ} \mathrm{C}\right.$, room temperature $(\mathrm{RT})=\sim 23$ ${ }^{\circ} \mathrm{C}$, and $40^{\circ} \mathrm{C}$ ) and monitored their time- and temperature-dependent color change. After one day, the THIs stored at higher temperature exhibited deeper color (Fig. 1b). The UV-Vis spectra show (Fig. 1c), as the storage temperature is increased from 0 to RT to $40^{\circ} \mathrm{C}$, the LSPR peak of AuNPs red-shifted from 555 to 559 to $569 \mathrm{~nm}$, along with increased absorbance. This suggests an increase in the size and number of AuNPs synthesized with increasing storage temperature (Sardar and Shumaker-Parry 2011; Wang and Gunasekaran 2012). 
After five days, the THIs stored at temperatures $0{ }^{\circ} \mathrm{C}$ and higher changed in color and intensity compared to their one-day stored counterparts (Figs. 1b and 1d), which implies that the alginate/AuNPs system can record not only temperature information, but also duration. The LSPR peak of AuNPs stored at $40^{\circ} \mathrm{C}$ blue-shifted from 568 to $537 \mathrm{~nm}$ (Figs. 1c and 1e), which typically correlates with changes in the morphology of AuNPs (discussed later).

The observed color change of the THIs after 14 days of storage at different temperatures is presented in Fig. 2a. We note here that the color of the THIs continued to change past 14 days. To evaluate the performance of THIs, their LSPR peak absorption data during the 14-day storage at RT were plotted against time as an example (Fig. 2 b). The linear correlation obtained $\left(\mathrm{R}^{2}=0.9756\right)$ confirms the color change and its predictability, given the thermal history. Fig. 2c shows the corresponding UV-Vis spectra of THIs at RT at each storage time. Therefore, the THIs can provide information that is visually discernable and readily traceable; moreover, the small error bars (i.e., standard deviations of three replicates) in Fig. 2b point to the reliability of the color change.

\subsection{Principles and potential mechanisms}

\subsubsection{Color-change mechanism}

According to the literature, the abundance of hydroxyl groups in carbohydrates can aid in efficient reduction reaction and stabilization of the particles within the matrix (Esumi et al. 2000; Saha et al. 2009). This suggests that sodium alginate (Fig. 3a) not only can reduce gold ions to gold atoms, but also can assist in stabilizing the AuNPs synthesized (Mele et al. 2014). The FT-IR spectra of 14-day stored samples (Fig. 3b) could help identify the functional groups of sodium alginate involved in the process. Fig. 3c displays that the THIs subjected to elevated temperatures showed decreasing strength of the band $\mathrm{v}(\mathrm{C}-\mathrm{OH})$ at $1408 \mathrm{~cm}^{-1}$ and $\mathrm{v}(\mathrm{CH}-\mathrm{OH})$ at $1123 \mathrm{~cm}^{-1}$ (Guo et al. 2009; Yang et al. 2007). The result implies the hydroxyl group could participate in the reduction of gold ions, concurring with previous studies (Esumi et al. 2000; Guo et al. 2009), and indicates that the reaction is strongly temperature dependent. That is, at higher temperatures the reduction of gold ions leads to the synthesis of more and smaller AuNPs, in addition, some of the smaller particles may attach on to the existing larger particles, which changes the LSPR and results in a more intense color. These results may explain why the THI color becomes more intense at higher temperatures. However, the reason for change in color at $40^{\circ} \mathrm{C}$ from deep grey to wine red over 14 days is not obvious. 
In addition, the XRD and XPS data (Fig. S2) also confirm the crystalline nature of the alginate/AuNPs. A typical XRD pattern of alginate with AuNPs grown in situ shows broad Bragg's reflections corresponding to $\{111\},\{200\},\{220\},\{311\}$ and $\{222\}$ lattice planes, suggesting the face-centered cubic (fcc) structure of AuNPs (Saha et al. 2009; Shankar et al. 2003). The broad peak at around $2 \theta=25^{\circ}$ indicates the high amorphous content of sodium alginate (Sarker et al. 2014).

The effect of storage time on the developed THI can be seen by comparing RT-stored THIs on days 0 and 14. Fig. S1a is the TEM image of as-synthesized THI that shows the initial size of AuNPs at RT, most of which are of diameter less than $200 \mathrm{~nm}$. The particle size increases with prolonged storage period at RT, resulting in increased particle diameters of around $300 \mathrm{~nm}$ shown in Fig. 4. The TEMs of samples stored for 14 days at RT show small particles have been synthesized and some of which are attached to the existing larger particles (Figs. $\mathbf{4 a}$ and $\mathbf{4 b}$ ), resulting in even larger particles, which is consistent with the speculation of the reduction of gold ions at elevated temperature is accentuated. (The white and grey areas in TEM images suggest the polymer structures of alginate under the electron beam.) However, large AuNPs are not seen in the TEM images of samples stored at $40^{\circ} \mathrm{C}$ (Fig. $\mathbf{4 c}$ and $\mathbf{4 d}$ ). A possible explanation is the increasing number of newly synthesized small AuNPs leads to lower solution viscosity such that the large AuNPs begin to settle down under the pull of gravity, especially when the newly synthesized AuNPs attach to them. Thus, only small AuNPs remain in the system. This hypothesis is corroborated by the grainy precipitate we observed at the bottom of the cuvettes.

\subsubsection{Viscosity change leads to additional color changes}

The above suggestion that the solution viscosity decreases with increasing number of newly in situ synthesized AuNPs is counter to Einstein-Batchelor law, according to which, in spherical particles suspensions, addition of particles always increases the fluid viscosity (Batchelor 1977; Einstein 1906). However, we confirmed a decrease in viscosity of alginate solutions in which AuNPs are synthesized in situ (Fig. S3a), which implies that viscosity of a biopolymer solution is reduced when nanoparticles are added to it. This is in line with a recent report that nanoparticle-polymer composites could exhibit unusual mechanical and dynamical features when the particles have at least one nanoscopic dimension, i.e., in the order of the chain dimensions of the host polymer (Jain et al. 2008; Mangal et al. 2015). In this case, the addition of nanoparticles could constrain the polymer chain motion, which eventually speeds up the relaxation of the polymer host. Several studies have shown similar results that the addition of unfunctionalized and 
polymer-functionalized nanostructures can produce conformational changes to polymer structures, which lowers the solution viscosity (Kalathi et al. 2012; Mackay et al. 2003; Tuteja et al. 2007).

Viscosity of alginate/AuNPs solution also decreased with time of storage at $40^{\circ} \mathrm{C}$ (Fig. $\mathbf{S 3 b}$ ). This lends support to our speculation above - i.e., as storage time at $40^{\circ} \mathrm{C}$ increased, more particles are synthesized in situ, which leads to viscosity reduction; meanwhile, some newly synthesized small AuNPs attach to existing larger particles (as observed in the TEM images) and, due to lower solution viscosity, begin to settle as a precipitate. Thus, the color of the system changes from deep grey to red, which is attributed to size-dependent changes in the LSPR.

We further investigated the effect of AuNPs on the sodium alginate solution viscosity using a highly concentrated alginate solution $\left(0.04 \mathrm{~g} \cdot \mathrm{mL}^{-1}\right)$ that promotes the synthesis of AuNPs. When heated at $90^{\circ} \mathrm{C}$, the color of this solution became deep grey in the first $30 \mathrm{~min}$ (Fig. 5a), suggesting an increase in the number of AuNPs, and then gradually turned red after $60 \mathrm{~min}$. These color changes are similar to those of alginate/AuNPs THIs stored at $40^{\circ} \mathrm{C}$.

In addition, as the heating continued, the system viscosity decreased (Fig. 5b), corresponding the color change, though we cooled all the samples similarly in an ice-water bath for $2.5 \mathrm{~min}$ before measurement. Further, the solution viscosity remained unchanged when measured without the in situ synthesis of AuNPs (Fig. 5c). Thus, the reduction of viscosity is most likely the result of increased number of AuNPs synthesized during heating. The TEM images confirm that the AuNPs were larger after heating for $30 \mathrm{~min}$ than for $60 \mathrm{~min}$ (Fig. S4a and S4b), consistent with those observed in the THI experiments above. In addition, when the samples obtained after $60 \mathrm{~min}$ heating were held at $40{ }^{\circ} \mathrm{C}$ for 30 days, most of the particles settled down and their color became transparent (Fig. 5d).

The results of this extended set of experiments confirm the precipitation of the AuNPs potentially due to viscosity reduction and suggest that increasing the concentration of alginate results in faster color change behavior, which implies that the alginate/AuNPs THIs are tunable. Besides, the THIs could also be used to monitor systems experiencing temperatures as high as 90 ${ }^{\circ} \mathrm{C}$. These results not only support the speculation that elevated temperatures facilitate the reduction of gold ion, but also potentially explain the reason of color change (from grey to wine red) as well as the particle sizes observed in the TEM images.

\subsection{THIs under temperature abuse scenario}


Generally, low-temperature storage is critical for safe storage of many bioproducts. For example, most commercial enzymes and antibodies are generally stored at $-20^{\circ} \mathrm{C}$. The quality of these perishable products is also extremely sensitive to temperature fluctuations. For instance, when stem cells are stored at RT overnight, their viability and recovery reduces significantly (Kelly et al. 2003). Therefore, we simulated a temperature abuse scenario by exposing frozen-stored samples to RT. We stored THIs in a freezer at $-20^{\circ} \mathrm{C}$ for $2 \mathrm{~h}$ and then exposed them to $\mathrm{RT}$ for 2 to $12 \mathrm{~h}$ before returning them to the freezer for additional 14 days.

Compared to the control (samples held frozen for 14 days without RT exposure), the THIs that experienced RT abuse exhibited a deeper color (Fig. S5a) and their corresponding UV-Vis spectra (Fig. S5b) exhibit higher absorption amplitude, which suggests that more AuNPs are present in the sample subjected to higher temperature (i.e., RT exposure). The UV-Vis spectrum was measured right after $1.5 \mathrm{~h}$ of thawing at RT. We also observed that the longer the temperature abuse, the deeper the color of the THIs (Fig. S6a). The total color difference (TCD) calculated using the $L^{*}, a^{*}, b^{*}$ system was used to determine the color change in the THIs exposed to different temperatures (Wanihsuksombat et al. 2010):

$$
T C D=\left[\left(\Delta L^{*}\right)^{2}+\left(\Delta a^{*}\right)^{2}+\left(\Delta b^{*}\right)^{2}\right]^{1 / 2}
$$

Where, $\Delta \mathrm{L}^{*}$ is the brightness difference, $\Delta \mathrm{a}^{*}$ is the redness-greenness difference, and $\Delta \mathrm{b}^{*}$ is the yellowness-blueness difference. The plot of TCD versus exposure time at RT was highly linear $\left(\mathrm{R}^{2}\right.$ $=0.9740)($ Fig. S6b). This result confirms that the THIs can be deployed to document the temperature history in a fairly predictable manner. More importantly, even if the products are returned to proper storage condition (i.e., freezer), any temperature abuse suffered can be elicited from the displayed color. These results suggest that the chemical reaction is irreversible, which is necessary for an indicator recording the product thermal history.

\subsection{Fabricating THI hydrogel}

One of the advantages of using alginate in THIs is its ability to form a hydrogel (Boland et al. 2007; Yang et al. 2011). Calcium and some other divalent cations can crosslink negatively charged alginic acid chains and form a well-known "egg-box" like structure (Fig. 6a) and solidify the matrix (Lee and Mooney 2012). Therefore, we introduced calcium ions into the alginate/AuNPs 
system. The THI hydrogels were subjected to temperature abuse as described above except that they were stored at $0{ }^{\circ} \mathrm{C}$ instead of at $-20^{\circ} \mathrm{C}$, and for 1 day instead of 14 days.

As discussed before, the THI hydrogel that experienced temperature abuse exhibited deeper color than the one that did not (Fig. 6b). Also, the longer the temperature abuse, the deeper the color of the THI hydrogel (Fig. S7a). The TCD of THIs versus RT exposure time was highly linear

$\left(\mathrm{R}^{2}=0.9513\right)$ (Fig. S7b), suggesting their potential traceability. Although the THI hydrogel may be readily implemented for practical applications, more systematic and detailed studies of the crystallinity of AuNPs throughout the temperature abuse process, the interaction between the hydrogel and the AuNPs structure should be investigated to understand their contributions to the temperature-dependent LSPR properties.

In practice, the alginate/AuNPs solution-based THIs can be contained in a transparent pouch of some sort, which is attached (or enclosed) to the package of container of products for the THIs to travel along with the products and track their thermal history. The hydrogel form of THI is easier to handle as they afford fabrication of rather solid-like tags, which can, however, be used as above. The color of the pouches or tags can be compared to a calibrated reference color strip developed for the particular THI system and an acceptable product thermal history to facilitate visual identification of any temperature abuse.

\subsection{Cost and Safety of the THIs}

The alginate/AuNPs THIs are cost-effective and environmentally benign. For hydrogel cubes (of volume $\sim 0.4 \mathrm{~mL}$ ) shown in Fig. 6c, the cost chemicals involved is $\sim \$ 2$ per 1,000 units (see Table S1 in Supporting Information for details). Alginate is safe and is used extensively in the pharmaceutical and food industries. In addition, AuNPs are non-cytotoxic and non-deleterious (Ghosh et al. 2008; Shukla et al. 2005) and are widely used in biological and biomedical applications. Therefore, our THI is a safe and inexpensive system for monitoring quality and safety of time- and temperature-sensitive food, biological, and pharmaceutical products.

\section{Conclusions}

We have developed a plasmonic THI using alginate-AuNP composite. By exploiting the LSPR changes of the evolving AuNPs, the THIs can be employed to track and document the thermal history by visually discernable color change. Our results show that the color of the THIs becomes more intense with increasing temperature and/or duration of storage. The decreasing 
viscosity with increasing number of AuNPs being synthesized in the system along with aggregation of newly synthesized AuNPs onto larger ones and their settling are potentially responsible for the distinct color change observed. The system is tunable by changing the THI composition and preparation conditions. In addition, using calcium ions, the alginate/AuNPs THI can be made in to a solid hydrogel, which may be more suitable for certain end-use applications. Overall the alginate/AuNPs THI is a simple, environmental-friendly, convenient, and inexpensive system to monitor quality and safety of bioproducts that are prone to time- and temperature-induced deterioration.

\section{Acknowledgment}

Y.-C. Wang acknowledges the support of Wisconsin Distinguished Graduate Fellowship. The authors acknowledge the use of facilities and instrumentation supported by the University of Wisconsin Materials Research Science and Engineering Center (DMR-1121288). This project was support by the USDA-NIFA Hatch grant (WIS01620).

\section{References}

Bagci, P.O., Wang, Y.-C., Gunasekaran, S., 2015. A Simple and Green Route for Room-Temperature Synthesis of Gold Nanoparticles and Selective Colorimetric Detection of Cysteine. Journal of Food Science 80(9), N2071-N2078.

Batchelor, G.K., 1977. The effect of Brownian motion on the bulk stress in a suspension of spherical particles. Journal of Fluid Mechanics 83(01), 97-117.

Boland, T., Tao, X., Damon, B.J., Manley, B., Kesari, P., Jalota, S., Bhaduri, S., 2007.

Drop-on-demand printing of cells and materials for designer tissue constructs. Materials Science and Engineering: C 27(3), 372-376.

Derwood, P., Aleksandar, M., 2007. Storage of hemopoietic stem cells. Asian J Transfus Sci. , 71-76.

Einstein, A., 1906. Zur Theorie der Brownschen Bewegung. Annalen der Physik 324(2), 371-381.

Esumi, K., Hosoya, T., Suzuki, A., Torigoe, K., 2000. Spontaneous Formation of Gold Nanoparticles in Aqueous Solution of Sugar-Persubstituted Poly(amidoamine)dendrimers. Langmuir 16(6), 2978-2980. 
Galliani, D., Mascheroni, L., Sassi, M., Turrisi, R., Lorenzi, R., Scaccabarozzi, A., Stingelin, N., Beverina, L., 2015. Thermochromic Latent-Pigment-Based Time-Temperature Indicators for Perishable Goods. Advanced Optical Materials 3(9), 1164-1168.

Ghosh, P., Han, G., De, M., Kim, C.K., Rotello, V.M., 2008. Gold nanoparticles in delivery applications. Advanced Drug Delivery Reviews 60(11), 1307-1315.

Guan, J., Wang, Y.-C., Gunasekaran, S., 2015. Using L-Arginine-Functionalized Gold Nanorods for Visible Detection of Mercury(II) Ions. Journal of Food Science 80(4), N828-N833.

Guo, R., Li, R., Li, X., Zhang, L., Jiang, X., Liu, B., 2009. Dual-Functional Alginic Acid Hybrid Nanospheres for Cell Imaging and Drug Delivery. Small 5(6), 709-717.

Heli, B., Morales-Narvaez, E., Golmohammadi, H., Ajji, A., Merkoci, A., 2016. Modulation of population density and size of silver nanoparticles embedded in bacterial cellulose via ammonia exposure: visual detection of volatile compounds in a piece of plasmonic nanopaper. Nanoscale 8(15), 7984-7991.

Jain, S., Goossens, J.G.P., Peters, G.W.M., van Duin, M., Lemstra, P.J., 2008. Strong decrease in viscosity of nanoparticle-filled polymer melts through selective adsorption. Soft Matter 4(9), 1848-1854.

Kalathi, J.T., Grest, G.S., Kumar, S.K., 2012. Universal Viscosity Behavior of Polymer Nanocomposites. Physical Review Letters 109(19), 198301.

Kelly, K.L., Coronado, E., Zhao, L.L., Schatz, G.C., 2003. The Optical Properties of Metal Nanoparticles: The Influence of Size, Shape, and Dielectric Environment. The Journal of Physical Chemistry B 107(3), 668-677.

Kim, K., Kim, E., Lee, S.J., 2012. New enzymatic time-temperature integrator (TTI) that uses laccase. Journal of Food Engineering 113(1), 118-123.

Lee, K.Y., Mooney, D.J., 2012. Alginate: Properties and biomedical applications. Progress in Polymer Science 37(1), 106-126.

Lim, S., Gunasekaran, S., Imm, J.Y., 2012. Gelatin-Templated Gold Nanoparticles as Novel Time-Temperature Indicator. Journal of Food Science 77(9), N45-N49.

Louis, I., Wagner, E., Dieng, M.M., Morin, H., Champagne, M.A., Haddad, E., 2012. Impact of storage temperature and processing delays on cord blood quality: discrepancy between functional in vitro and in vivo assays. Transfusion 52(11), 2401-2405. 
Lu, L., Seenivasan, R., Wang, Y.-C., Yu, J.-H., Gunasekaran, S., 2016. An Electrochemical Immunosensor for Rapid and Sensitive Detection of Mycotoxins Fumonisin B1 and Deoxynivalenol. Electrochimica Acta 213, 89-97.

Mackay, M.E., Dao, T.T., Tuteja, A., Ho, D.L., Van Horn, B., Kim, H.-C., Hawker, C.J., 2003. Nanoscale effects leading to non-Einstein-like decrease in viscosity. Nat Mater 2(11), 762-766. Mangal, R., Srivastava, S., Archer, L.A., 2015. Phase stability and dynamics of entangled polymer-nanoparticle composites. Nat Commun 6.

Mele, E., Anyfantis, G.C., Fragouli, D., Ruffilli, R., Athanassiou, A., 2014. Localized synthesis of gold nanoparticles in anisotropic alginate structures. RSC Advances 4(39), 20449-20453. Pereira Jr, V.A., de Arruda, I.N.Q., Stefani, R., 2015. Active chitosan/PVA films with anthocyanins from Brassica oleraceae (Red Cabbage) as Time-Temperature Indicators for application in intelligent food packaging. Food Hydrocolloids 43, 180-188.

Saha, S., Pal, A., Pande, S., Sarkar, S., Panigrahi, S., Pal, T., 2009. Alginate Gel-Mediated Photochemical Growth of Mono- and Bimetallic Gold and Silver Nanoclusters and Their Application to Surface-Enhanced Raman Scattering. The Journal of Physical Chemistry C 113(18), 7553-7560.

Sardar, R., Shumaker-Parry, J.S., 2011. Spectroscopic and microscopic investigation of gold nanoparticle formation: ligand and temperature effects on rate and particle size. J. Am. Chem. Soc. 133(21), 8179-8190.

Sarker, B., Papageorgiou, D.G., Silva, R., Zehnder, T., Gul-E-Noor, F., Bertmer, M., Kaschta, J., Chrissafis, K., Detsch, R., Boccaccini, A.R., 2014. Fabrication of alginate-gelatin crosslinked hydrogel microcapsules and evaluation of the microstructure and physico-chemical properties. Journal of Materials Chemistry B 2(11), 1470-1482.

Shankar, S.S., Ahmad, A., Pasricha, R., Sastry, M., 2003. Bioreduction of chloroaurate ions by geranium leaves and its endophytic fungus yields gold nanoparticles of different shapes. Journal of Materials Chemistry 13(7), 1822-1826.

Shukla, R., Bansal, V., Chaudhary, M., Basu, A., Bhonde, R.R., Sastry, M., 2005.

Biocompatibility of Gold Nanoparticles and Their Endocytotic Fate Inside the Cellular Compartment: A Microscopic Overview. Langmuir 21(23), 10644-10654.

Taoukis, P.S., Labuza, T.P., 1989. Applicability of Time-Temperature Indicators as Shelf Life Monitors of Food Products. Journal of Food Science 54(4), 783-788. 
Tuteja, A., Duxbury, P.M., Mackay, M.E., 2007. Multifunctional Nanocomposites with Reduced Viscosity. Macromolecules 40(26), 9427-9434.

Wang, S., Liu, X., Yang, M., Zhang, Y., Xiang, K., Tang, R., 2015a. Review of Time

Temperature Indicators as Quality Monitors in Food Packaging. Packaging Technology and Science 28(10), 839-867.

Wang, Y.-C., Cokeliler, D., Gunasekaran, S., 2015b. Reduced Graphene Oxide/Carbon

Nanotube/Gold Nanoparticles Nanocomposite Functionalized Screen-Printed Electrode for Sensitive Electrochemical Detection of Endocrine Disruptor Bisphenol A. Electroanalysis 27(11), 2527-2536.

Wang, Y.-C., Gunasekaran, S., 2012. Spectroscopic and microscopic investigation of gold nanoparticle nucleation and growth mechanisms using gelatin as a stabilizer. J Nanopart Res 14(10), 1-11.

Wang, Y.-C., Lu, L., Gunasekaran, S., 2015c. Gold nanoparticle-based thermal history indicator for monitoring low-temperature storage. Microchimica Acta 182(7-8), 1305-1311.

Wanihsuksombat, C., Hongtrakul, V., Suppakul, P., 2010. Development and characterization of a prototype of a lactic acid-based time-temperature indicator for monitoring food product quality. Journal of Food Engineering 100(3), 427-434.

Wei, L., Wang, X., Li, C., Li, X., Yin, Y., Li, G., 2015. Colorimetric assay for protein detection based on "nano-pumpkin" induced aggregation of peptide-decorated gold nanoparticles. Biosensors and Bioelectronics 71, 348-352.

Xu, M., Wang, X., Yan, Y., Yao, R., Ge, Y., 2010. An cell-assembly derived physiological 3D model of the metabolic syndrome, based on adipose-derived stromal cells and a gelatin/alginate/fibrinogen matrix. Biomaterials 31(14), 3868-3877.

Yang, H., Yan, R., Chen, H., Lee, D.H., Zheng, C., 2007. Characteristics of hemicellulose, cellulose and lignin pyrolysis. Fuel 86(12-13), 1781-1788.

Yang, J.-S., Xie, Y.-J., He, W., 2011. Research progress on chemical modification of alginate: A review. Carbohydrate Polymers 84(1), 33-39.

Zhang, C., Yin, A.-X., Jiang, R., Rong, J., Dong, L., Zhao, T., Sun, L.-D., Wang, J., Chen, X., Yan, C.-H., 2013. Time-Temperature Indicator for Perishable Products Based on Kinetically Programmable Ag Overgrowth on Au Nanorods. ACS Nano 7(5), 4561-4568. 
Zhang, H., Ma, X., Liu, Y., Duan, N., Wu, S., Wang, Z., Xu, B., 2015. Gold nanoparticles enhanced SERS aptasensor for the simultaneous detection of Salmonella typhimurium and Staphylococcus aureus. Biosensors and Bioelectronics 74, 872-877.

Zheng, J.Y., Janis, L.J., 2006. Influence of $\mathrm{pH}$, buffer species, and storage temperature on physicochemical stability of a humanized monoclonal antibody LA298. International Journal of Pharmaceutics 308(1-2), 46-51.

\section{Figures}

\section{Figure 1}

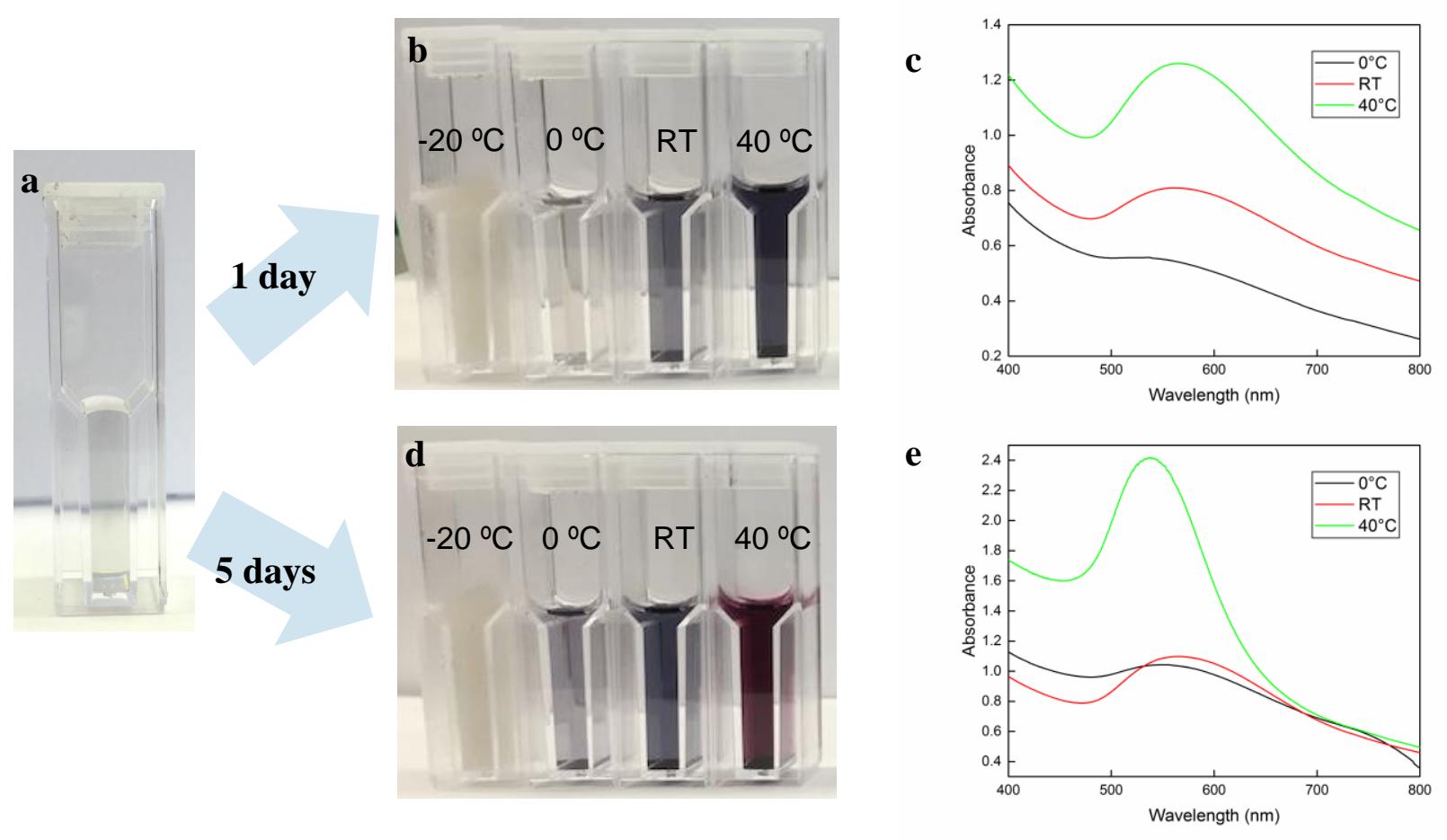


Figure 2
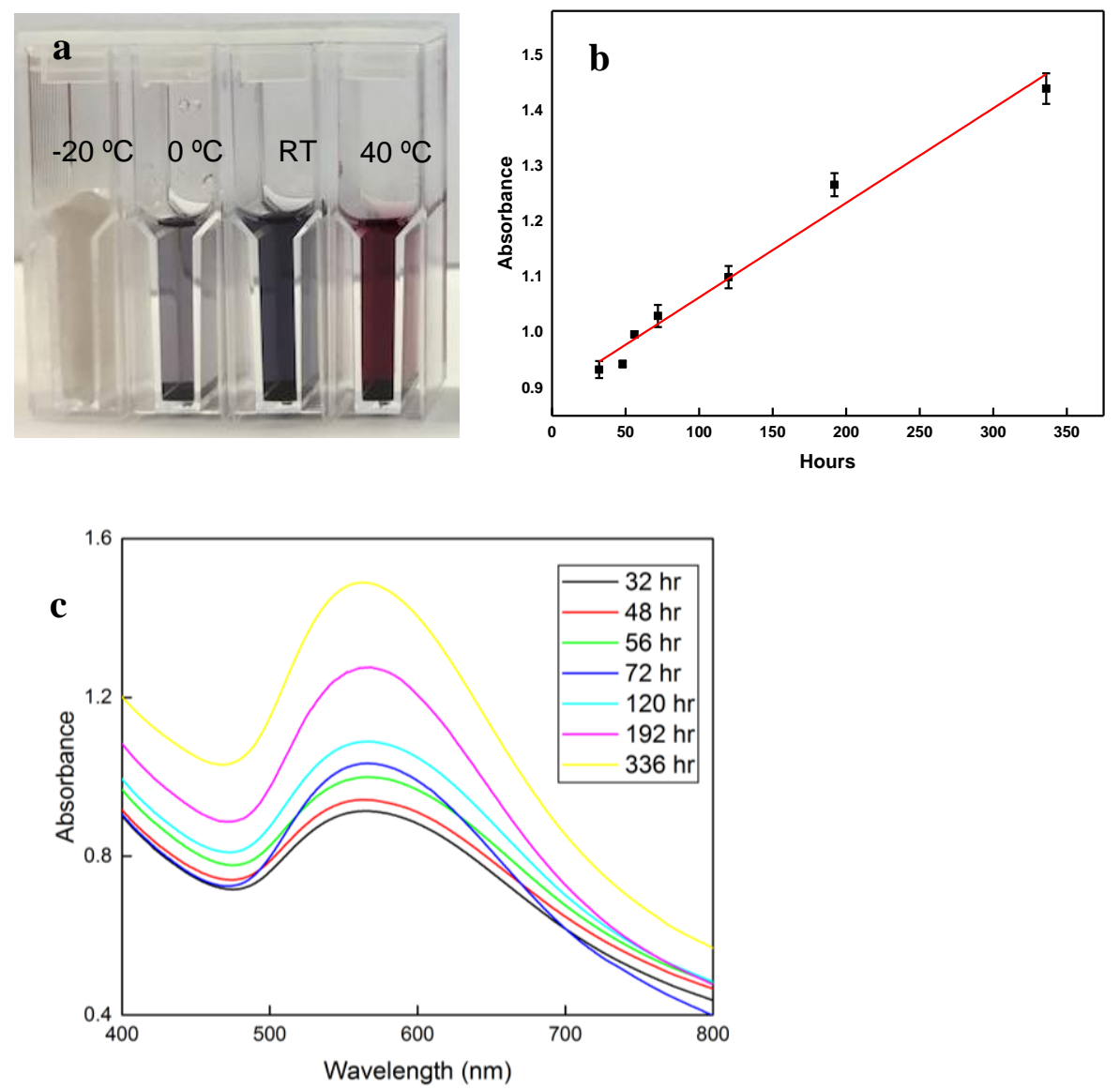
Figure 3

a

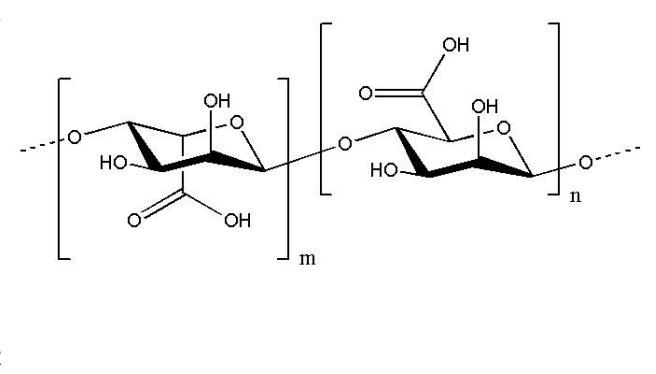

b
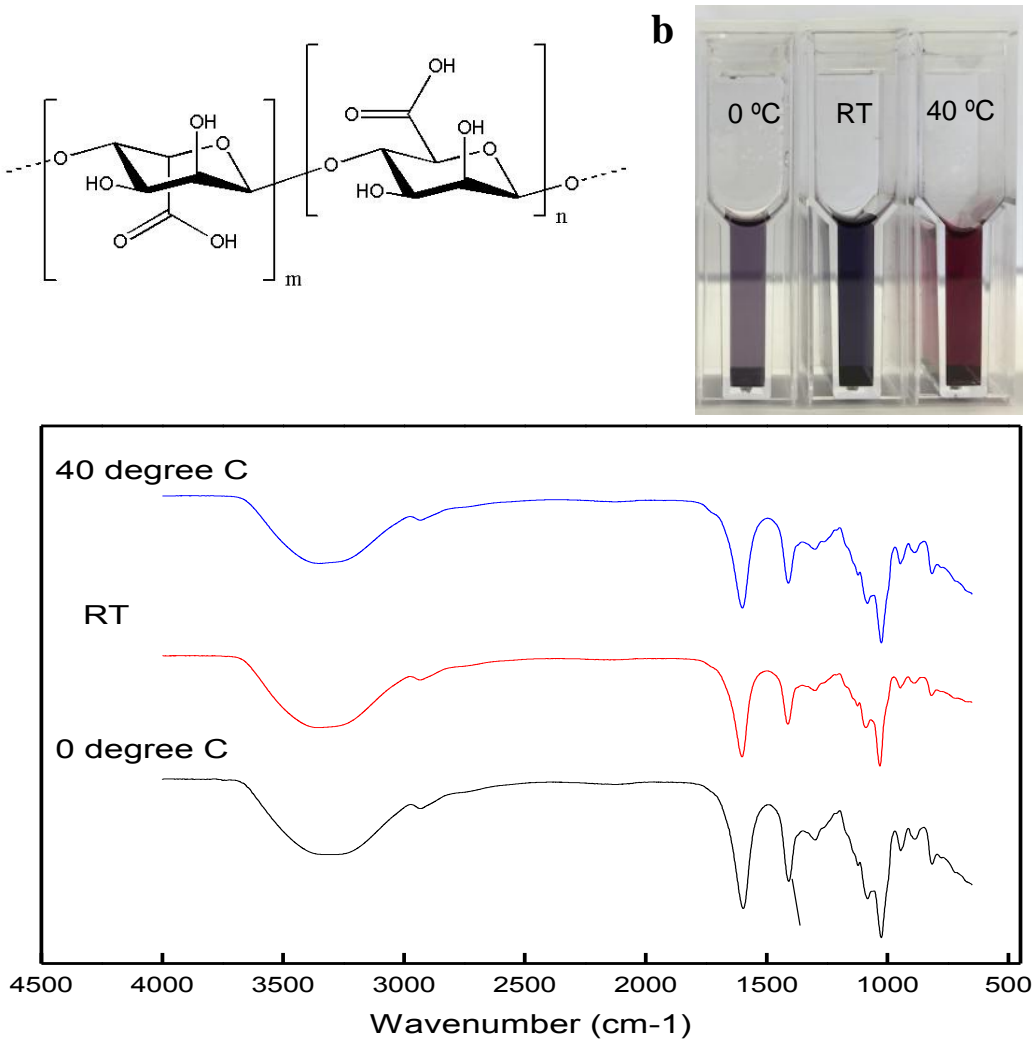
Figure 4

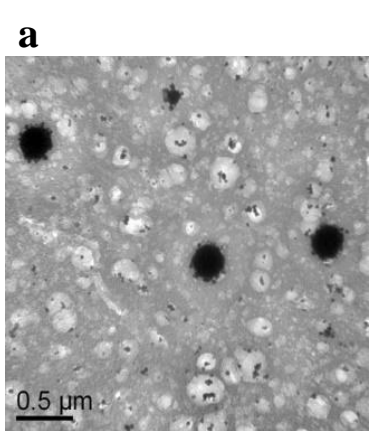

b
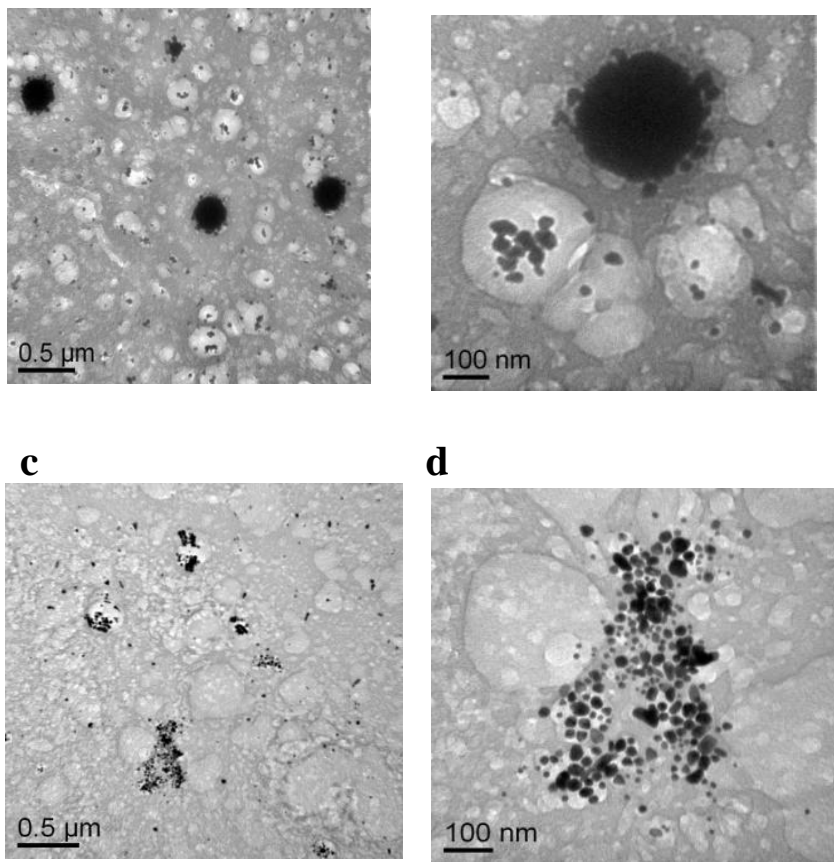

d

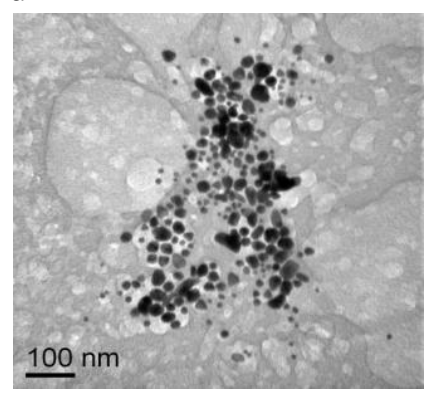


Figure 5

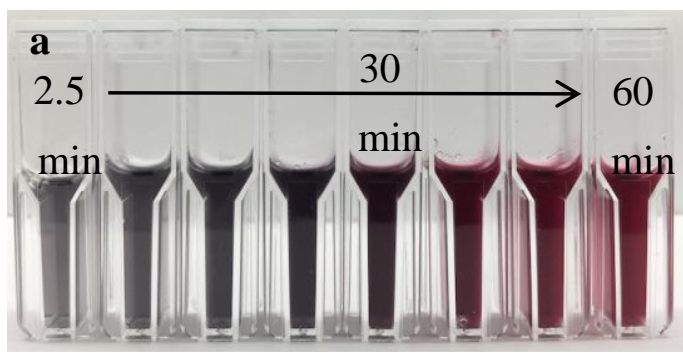

d

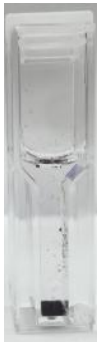

b

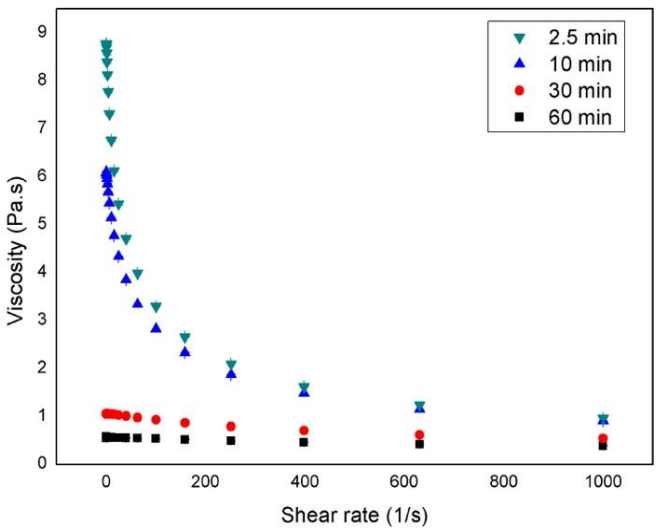

c

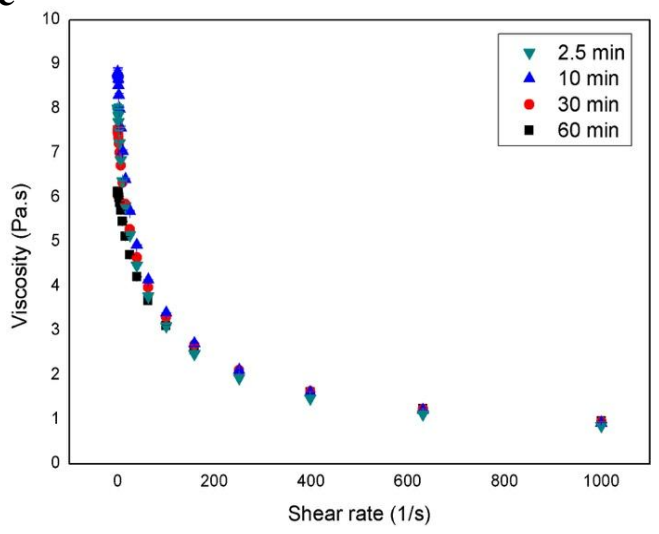


Figure 6
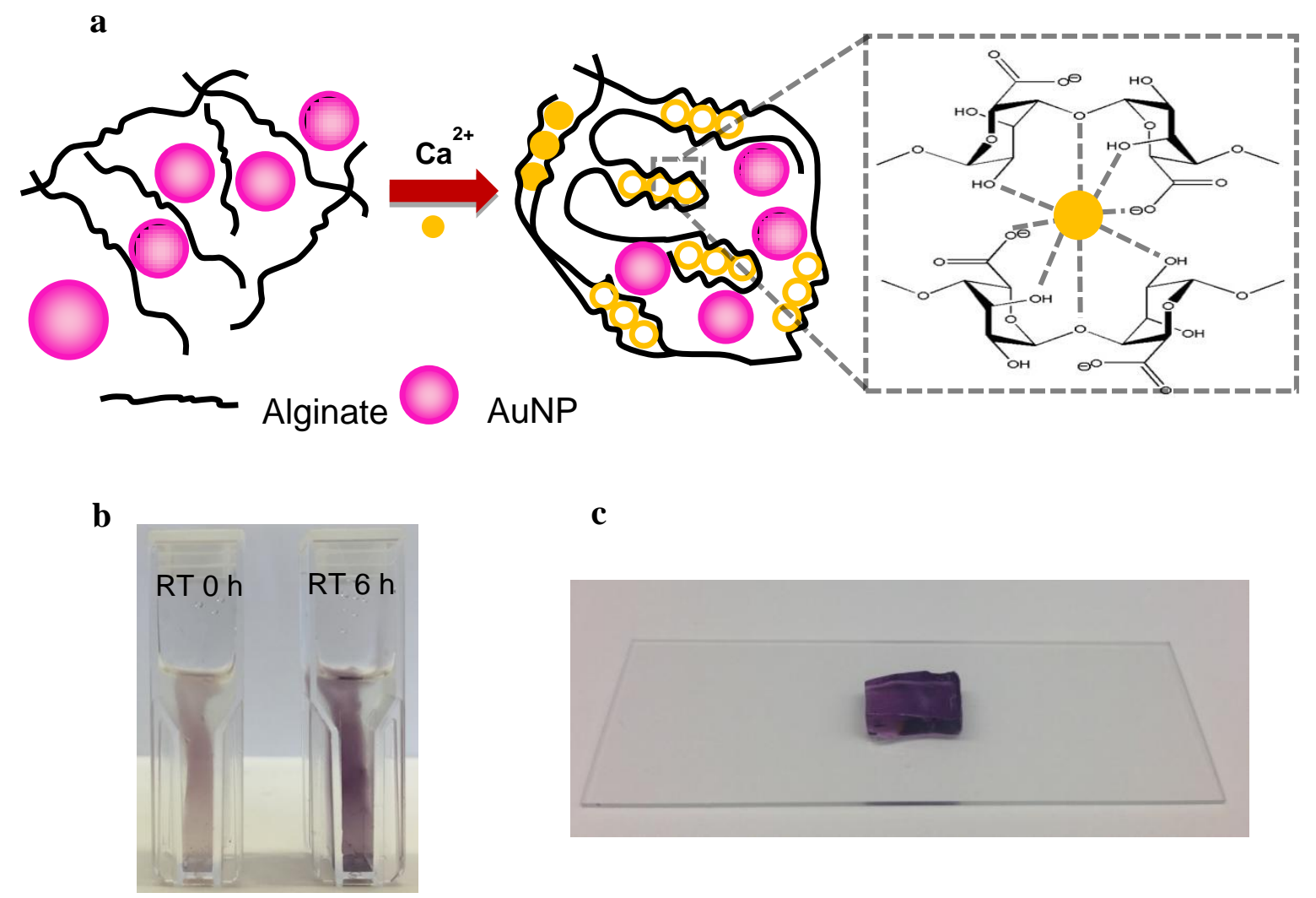


\section{Figure Captions}

Figure 1. (a) as-prepared alginate/AuNPs THI. Color (b and d) and UV-Vis spectra (c and e) of THIs stored at different temperatures after 1 day and 5 days. THIs were prepared by mixing $1 \mathrm{mM}$ of $\mathrm{HAuCl}_{4}$ with $0.01 \mathrm{~g} \cdot \mathrm{mL}^{-1}$ sodium alginate solution and heating the mixture at $90{ }^{\circ} \mathrm{C}$ for $2.5 \mathrm{~min}$.

Figure 2. (a) Color of THIs after 14-day storage at different temperatures and (b) peak amplitude of UV-Vis spectra vs. time of storage at room temperature (RT). (c) UV-Vis spectra of THIs being stored at RT for different durations. THIs were prepared by mixing $1 \mathrm{mM}$ of $\mathrm{HAuCl}_{4}$ with 0.01 $\mathrm{g} \cdot \mathrm{mL}^{-1}$ sodium alginate solution and heating the mixture at $90^{\circ} \mathrm{C}$ for $2.5 \mathrm{~min}$.

Figure 3. (a) Chemical structure of alginic acid. (b) Color and (c) FT-IR spectra of alginate/ AuNPs THIs stored for 14 days at different temperatures. THIs were prepared by mixing $1 \mathrm{mM}$ of $\mathrm{HAuCl}_{4}$ with $0.01 \mathrm{~g} \cdot \mathrm{mL}^{-1}$ sodium alginate solution and heating the mixture at $90^{\circ} \mathrm{C}$ for $2.5 \mathrm{~min}$.

Figure 4. TEM images of alginate/AuNPs THIs after 14 days $(\mathbf{a}, \mathbf{b})$ at room temperature and $(\mathbf{c}, \mathbf{d})$ at $40^{\circ} \mathrm{C}$.

Figure 5. The color (a) and viscosity (b) of the alginate/AuNPs system, with $0.04 \mathrm{~g} \cdot \mathrm{mL}^{-1}$ of alginate, after heating at $90{ }^{\circ} \mathrm{C}$ for 2.5 to $60 \mathrm{~min}$. (c) viscosity of $0.04 \mathrm{~g} \cdot \mathrm{mL}^{-1}$ of alginate after heating at $90{ }^{\circ} \mathrm{C}$ for 2.5 to $60 \mathrm{~min}$. (d) color of the THI obtained after $60 \mathrm{~min}$ heating (the right most in a) disappears when held at $40{ }^{\circ} \mathrm{C}$ for 30 days, but a dark discoloration at the bottom indicates that AuNPs have settled down.

Figure 6. (a) Scheme for alginate/AuNPs hydrogel formation with the addition of calcium ions. (b) Alginate/AuNPs THI hydrogels stored at $0{ }^{\circ} \mathrm{C}$ for $2 \mathrm{~h}$ and exposure to room temperature (RT) for 0 and $6 \mathrm{~h}$ followed by storage at $0^{\circ} \mathrm{C}$ for $24 \mathrm{~h}$. (c) Alginate/AuNPs THI hydrogel. 